\title{
LOS JAPONESISMOS RELIGIOSO-FILOSÓFICOS EN EL ESPAÑOL ACTUAL
}

\section{JAPANESE RELIGIOUS AND PHILOSOPHICAL LOANWORDS IN CURRENT SPANISH}

\author{
RAFAel Fernández Mata \\ Universidad de Córdoba \\ rafaelfernandezmata@gmail.com
}

RESUmen: En el inventario de japonesismos usados en el español actual, las voces de origen japonés relacionadas con la religión y la filosofía constituyen la séptima mayor área referencial -posición que comparten también con los japonesismos relativos a la vivienda-mobiliario y a la vestimenta- (4 voces de un total de 92 ítems). En el presente artículo se intenta, por primera vez en la lengua española, llevar a cabo un estudio exhaustivo de los japonesismos religioso-filosóficos, atendiendo a parámetros lingüísticos diferentes (formal, semántico e histórico), además de indicar su grado de adaptación al inventario léxico del español.

Palabras clave: japonesismo; religión; filosofía; préstamos léxicos; lexicografía española.

Abstract: Japanese loanwords relating to religion and philosophy constitute the seventh biggest semantic field -a position that they also share with Japanese loanwords related to home-furniture and clothing- of all such words used currently in Spanish (4 words out of a total of 92). In the present article an attempt is made for the first time in Spanish to offer an exhaustive description of religious and philosophical Japanese loanwords from a formal, semantic and historical perspective. The degree to which these loanwords adapt to the Spanish lexicon is also indicated.

Keywords: japonesismo; religion; philosophy; loanwords; Spanish lexicography.

Recepción: 7 de septiembre de 2017; aceptación: 24 de abril de 2018.

D.R. () 2019. Nueva Revista de Filología Hispánica Licencia Creative Commons Attribution-NonCommercial (CC BY-NC) 4.0 International 


\section{INTRODUCCIÓN}

En la lexicología actual resulta comúnmente aceptada la idea de que las lenguas enriquecen su acervo léxico con elementos tomados en préstamo de otros sistemas (Martinet 1991 [1965], p. 216; Weinreich 1974 [1968], p. 107; Robins 1995 [1971], p. 511; Sala 1998 [1986], p. 229; García Yebra 1994, p. 279; Gómez Capuz 1998, p. 13). Tal es el caso de la lengua española, cuyos préstamos suponen el $41 \%$ del total de sus unidades léxicas (Alvar 2012 [1994], p. 10).

A pesar de este elevado porcentaje de voces tomadas de otros sistemas lingüísticos, muy pocos investigadores han indagado la parte de ese $41 \%$ perteneciente al léxico procedente de la lengua japone$\mathrm{sa}^{1}$, el cual, por lo demás, no resulta tan elevado si lo comparamos con los préstamos de otras lenguas ${ }^{2}$.

Por lo que respecta al inventario de voces tomadas del japonés, o japonesismos $^{3}$, en nuestra tesis inédita (Los japonesismos de la lengua española: historia y transcripción; véase Fernández Mata 2015) y en el artículo titulado "Los japonesismos del español actual" (Fernández Mata, 2017) establecimos un inventario de 92 ítems que son utilizados en la lengua española actual -entendida ésta desde una visión panhispánica.

Aplicamos una serie de parámetros semánticos a dicho inventario y dividimos las unidades según 12 áreas referenciales, las cuales, de mayor a menor, son las siguientes: artes marciales y deporte (18 unidades); cocina y alimentación (16); guerra y gobierno de la nación (10); artes (8); ocio (7); escritura y literatura (6); vivienda y mobiliario (4); vestimenta (4); filosofía y religión (4); botánica (3); economía (3); medicina (3). Adviértase, pues, que en este artículo se analizarán las unidades relativas a una de las tres áreas referenciales (la vivienda-mobiliario, la vestimenta y lo religioso-filosófico) que componen el séptimo mayor grupo de voces procedentes de la lengua japonesa, es decir, nos ocuparemos del estudio exhaustivo de cuatro

${ }^{1}$ Hay varios trabajos en los que se ofrece un inventario -en ningún caso exhaustivo- de japonesismos: Knowlton 1959; Kim 1992; Frago Gracia 1997; Gómez Capuz 2004; Reyes Díaz 2004; Prieto 2007; Cid Lucas 2009; Giménez Folqués 2012. También encontramos una breve referencia a varios japonesismos introducidos por medio de la lengua portuguesa en el capítulo de "Lusismos" de la Enciclopedia lingüistica hispánica.

${ }^{2}$ Por ejemplo, según el Diccionario de uso del español de América y España (2011), el español cuenta con 324 voces procedentes de la lengua francesa y 422 de la lengua inglesa, mientras que en el caso de la lengua japonesa, sólo se recogen 44 lemas.

${ }^{3}$ En el artículo "Etiqueta para denominar los préstamos léxicos de la lengua japonesa: japonesismos" pedimos a los investigadores que utilizaran dicho término para designar las voces tomadas en préstamo de la lengua japonesa y así evitar la confusión con otro "sinónimo", japonismo, que podría especializarse para referirse a la influencia cultural japonesa en Occidente, sobre todo pictórica. 
voces pertenecientes a la religión y la filosofía: «bonzo», «cen», «sintoísmo»y «zacén» ${ }^{4}$.

Para el análisis de estos japonesismos, el artículo se organizará en tres grandes apartados. En el epígrafe de metodología se detallan las fuentes empleadas para reunir toda la información relativa a estas voces $^{5}$. Contiene, asimismo, un subapartado en el que explicamos nuestra concepción sobre las etapas de los préstamos. En el tercer punto se realiza el análisis lingüístico de las voces seleccionadas. En último lugar, se exponen las conclusiones de nuestra investigación.

\section{Metodología}

Con el objeto de recabar el mayor número de datos posible acerca de la expresión, el contenido, la etimología y la historia de los japonesismos religioso-filosóficos, tuvimos en cuenta diversos materiales. Por un lado, para la descripción fonético-gráfica, morfosintáctica y semántica, recurrimos a las muestras en los corpus de referencia del español (el CORDE, el CREA y el CORPES XXI) ${ }^{6}$; por otro, buscamos información en las siguientes obras lexicográficas hispánicas, todas de corte sincrónico: DVUA, DEA, DLE 2001 -vigésima segunda edición-, GDUEA, DUEAE, NDVUA, DUE, DClave y DLE 2014 -vigésima tercera edición. Además, intentamos perfeccionar nuestra descripción examinando de cerca otros diccionarios de lenguas europeas: $M W C D$ y $O E D$ (inglés), DFL y $L P R$ (francés), DHLP y DPLP (portugués) y

${ }^{4} \mathrm{Al}$ igual que en nuestra tesis inédita, en todas aquellas voces de origen japonés que siguen nuestro sistema de transcripción al alfabeto latino, emplearemos comillas latinas («»), y cursivas para aquellas voces que todavía se consideran extranjerismos. Para conocer nuestros patrones transcriptivos, cf. Fernández MATA 2018a. A lo largo del presente estudio hemos transcrito todas las voces de origen japonés (nombres propios, obras lexicográficas, ciudades, etc.) según nuestro método de transcripción, con la única excepción de los nombres propios de los autores que hemos consultado para nuestra bibliografía.

${ }_{5}^{5}$ Puesto que no forma parte de los objetivos de este trabajo, no profundizaremos en cuáles han sido los pasos que hemos seguido para obtener el listado de 92 unidades procedentes de la lengua japonesa. No obstante, a modo de resumen, nuestro proceso consistió en cruzar la información de dos fuentes: en primer lugar, recurrimos a distintos portales cibernéticos (Google, Dialnet, Academia.edu, Biblioteca Miguel de Cervantes e Infoling) para comprobar si previamente se había compilado un listado de japonesismos. Sumamos las unidades encontradas en estas primeras fuentes a los inventarios de japonesismos que reunían 9 obras lexicográficas de corte panhispánico -utilizadas en este artículo- y obtuvimos un inventario de 148 voces. Aplicamos una serie de filtros a dicha lista con el fin de evitar repeticiones, ítems de origen no japonés y vocablos cuyo uso era nulo, muy restringido o circunstancial, lo que nos llevó a las 92 unidades. Para una explicación más detallada, cf. Fernández MATA 2015 y 2017.

${ }^{6}$ Para las abreviaturas usadas en este artículo, véase el Anexo que ofrecemos al final. 
DOVLI y Zingarelli (italiano). Por lo que se refiere al análisis histórico-etimológico de las palabras, empleamos: 1) dos obras españolas fundamentales para el estudio diacrónico (el DCELC y el buscador en línea del NTLLE); 2) la información etimológica que reflejan los diccionarios hispánicos (de los nueve descritos, sólo tomaremos el DLE 2001, el GDUEA, el DUEAE, el DUE, el DClave y el DLE 2014, ya que son los únicos que incluyen el estudio etimológico); 3) los descriptores etimológicos de los diccionarios extranjeros; 4) dos obras lexicográficas japonesas (el DaiD y el Daiyirín).

En cuanto al análisis de los japonesismos religioso-filosóficos, hemos repartido la información obtenida en las fuentes anteriores en 7 párrafos, cada uno relacionado con diversos parámetros:

Primer párrafo: cuestiones relativas a la etimología. Tratamos de despejar las siguientes incógnitas: 1) étimo japonés del que procede y pronunciación japonesa del mismo; 2) categoría gramatical del étimo y acepciones de éste en lengua japonesa.

Segundo, tercer y cuarto párrafos: datos sobre la expresión, el contenido y el uso de un determinado japonesismo, respectivamente. En el segundo párrafo, dedicado a la forma gráfica y a la acentuación, respondemos a preguntas como: 1) forma y variantes gráficas con que ha sido adaptada tal palabra (¿patrón gráfico español, extranjerizante o híbrido?); 2) ¿influjo o procedencia de otra lengua intermediaria? En el tercer parágrafo, donde se examinan el significado y el área referencial, despejamos las siguientes interrogantes: 1) acepciones en español (¿coincide con el japonés u otra lengua intermediaria?); 2) área referencial. En el cuarto y último apartado, donde se estudian las características morfosintácticas, analizamos: 1) género y número (masculino/ femenino, singular/ plural); 2) patrones combinatorios (uso con artículo, adjetivos, adverbios, etcétera).

Quinto, sexto y séptimo párrafos: información histórica del vocablo. En el quinto párrafo resolvemos: 1) ¿cuándo se tiene constancia del primer registro escrito en lengua española? Bien sea textual (proporcionado por los corpus CORDE, CREA y CORPES XXI), bien lexicográfico (conseguido en las obras lexicográficas de carácter histórico: DCELC y NTLLE; o actual: DVUA, DEA, DLE 2001, GDUEA, DUEAE, NDVUA, DUE, DClave y DLE 2014); 2) ¿se observa algún tipo de variación formal o significativa desde su primer registro? En el sexto párrafo, revisamos brevemente la primera documentación de un japonesismo en las otras lenguas extranjeras y comparamos estos datos con los obtenidos en las obras hispánicas. En el séptimo y último párrafo, se concluye el estudio con el análisis relativo al fenómeno del préstamo léxico, es decir, valoramos en qué momento de adaptación se encuentra un japonesismo en particular (cf. el siguiente apartado). Determinamos, asimismo, su repercusión en el sistema léxico del español. 
Pese a que compartimos la opinión de Gómez Capuz (1998, p. 214) de que resulta muy complejo diseccionar o "acotar en etapas discretas lo que parece ser un verdadero «continuum» entre el extranjerismo crudo y ocasional y el préstamo usual y perfectamente adaptado", nos basamos en su clasificación tripartita para analizar el momento en que se encuentra un determinado japonesismo: el momento de transferencia, el momento de asimilación y el momento de madurez (Gómez Capuz 1998, p. 219; 2005, p. 15).

La primera etapa o momento de transferencia de un préstamo léxico se caracteriza por el uso limitado del vocablo en cuestión, que queda relegado al lenguaje o campo técnico con el que esté relacionado. En lo concerniente a su aspecto formal, la voz en préstamo entra con su grafía y sonido originales (es precisamente este rasgo gráfico el que desechamos para nuestro modelo teórico, dado que la lengua japonesa no utiliza el alfabeto latino). Tras verse desvinculada de los paradigmas formales nativos, se observa inestabilidad y anarquía formal en la voz que se ha tomado en préstamo, que puede sufrir cambios fonéticos y gráficos esporádicos. Por lo que se refiere al componente semántico, la monosemia regula el proceso, ya que el vocablo es adquirido con un solo uso denotativo y libre de cualquier paradigma semántico o asociación emotiva. Debido a la novedad del término, suele ir acompañado por marcas autonímicas como sinónimos, paráfrasis o explicaciones para su comprensión (Gómez Capuz 1998, pp. 220-222; 2005, pp. 15-16, 29).

En cuanto a la segunda etapa o proceso de asimilación, el extranjerismo demuestra su eficacia neológica y comienza un proceso de integración en el nuevo sistema que puede prolongarse en el tiempo y supone una evolución de estatus de un determinado préstamo léxico: desde su condición de extranjerismo hasta llegar a ser préstamo, cuando ya se ha asimilado formalmente, es ampliamente utilizado por muchos hablantes de la lengua receptora, quienes ya apenas lo perciben como elemento exógeno. El proceso de adaptación o naturalización de un determinado extranjerismo supone la asimilación de éste a todos los subsistemas o niveles de análisis de la lengua receptora: por lo que se refiere a los niveles fónicográfico y morfológico, el préstamo léxico se adecua paulatinamente a los patrones gráficos de la lengua española y modifica ciertas realizaciones extranjerizantes para adaptarlas al de los sonidos de la lengua de destino -una asimilación fónica-; asimismo, adquiere los rasgos morfológicos del español (como el género, el número, etc.). Además, en cuanto al nivel semántico, suele dejar atrás la monosemia para integrarse en las estructuras lexemáticas o área referencial de la lengua receptora. En ocasiones, cuando se trata 
de términos técnicos, se produce una simple adición al inventario léxico del español, mientras que en otras, cuando el extranjerismo tiene ciertos valores semánticos con palabras nativas, se puede producir la especialización semántica del término (Gómez Capuz 1998, pp. 223-251; 2005, pp. 17-25).

En la tercera etapa o etapa de explotación, madurez y creatividad, el préstamo lingüístico se caracteriza por estar completamente asimilado en todos los niveles, por lo que se percibe como elemento patrimonial de la lengua receptora. De este modo, queda sometido a la acción dinámica, creativa y neológica del lenguaje, es decir, comienza a generar nuevos elementos como si se tratara de un recurso neológico interno, y sigue, en consecuencia, mecanismos morfosintácticos (formación de derivados, compuestos híbridos, alteración de compuestos nominales y cambio de categoría gramatical) y semánticos (generalización, metáfora, metonimia) propios de la lengua receptora (Gómez Capuz 1998, pp. 251-254; 2005, pp. 25-27).

Por lo que respecta al uso general de las palabras analizadas, comprobaremos la frecuencia normalizada de éstas tanto en la versión anotada del CREA como en el CORPES XXI. No sólo examinaremos la evolución de su uso, sino también la distribución geográfica de la voz y la tipología textual en que tiene lugar. Ya que pretendemos conseguir una cifra lo más ajustada posible a la realidad, introduciremos en el motor de búsqueda de las plataformas el significante singular de las voces sin marcar la casilla de "grafía original", para permitir que éstos consideren también las posibles variantes gráficas de un japonesismo -por ejemplo, con o sin tilde, con o sin mayúscula inicial, etc. Para establecer una escala, usaremos los parámetros desarrollados por el GDUEA ${ }^{7}$, esto es, las marcas de frecuencia se atienen a los siguientes porcentajes: 1) frecuencia circunstancial o no significativa

7 Esta obra lexicográfica fue elaborada a partir de los ejemplos de uso proporcionados por un corpus de 20 millones de palabras. El corpus utilizado como base es ampliamente representativo de la lengua española en España e Hispanoamérica (sin olvidar las áreas hispanohablantes de Estados Unidos), en sus variedades escrita y oral, y en géneros y ámbitos variados, tal cual se describe en la obra de Aquilino Sánchez et al., Cumbre. Corpus lingüístico del español contemporáneo (1995). Por lo que se refiere a su frecuencia de uso, leemos en la descripción aportada por el GDUEA: "Para alcanzar un razonable índice de fiabilidad en estas marcas de frecuencia, se han llevado a cabo determinados cálculos estadísticos relativos al total de formas y palabras presentes en el Corpus Cumbre, a los tramos de frecuencia de las más de 250000 formas diferentes que registra dicho corpus y a la cantidad de formas abarcadas por cada tramo. Cabe señalar que en torno al $50 \%$ de las voces marcadas pertenece al nivel de «Frecuencia baja» y que el umbral fijado para que una voz acceda al tramo más bajo de frecuencia ha sido de cinco ocurrencias sobre el total del corpus (equivalentes al 0.25 por millón). Por debajo de este umbral, y en relación con el corpus manejado (de 20 millones de palabras), 
(hasta 0.25 por millón); 2) frecuencia baja (desde 0.26 por millón hasta 2.99 por millón); 3) frecuencia moderada (desde 3 hasta 10.99 por millón); 4) frecuencia notable (desde 11 hasta 25.99 por millón); 5) frecuencia alta (desde 26 hasta 75 por millón); 6) frecuencia muy alta (más de 75 por millón).

Por tanto, consideraremos todas estas variables para adscribir un determinado japonesismo a un grupo en particular: el de las voces recién transferidas ${ }^{8}$, el de las voces en proceso de asimilación y el de las voces patrimoniales. Al final de cada estudio pormenorizado de los japonesismos, añadiremos un párrafo en que se ofrecerá un razonamiento detallado que describa las causas por las que tal japonesismo queda incluido en determinado grupo.

\section{TABLA 1}

Parámetros con que determinar el momento de adopción de un préstamo

\begin{tabular}{|c|c|c|}
\hline $\begin{array}{l}1^{a} \text { etapa } \\
\text { Momento de transferencia }\end{array}$ & $\begin{array}{l}2^{a} \text { etapa } \\
\text { Momento de asimilación }\end{array}$ & $\begin{array}{l}3^{a} \text { etapa } \\
\text { Momento de madurez }\end{array}$ \\
\hline $\begin{array}{l}\text { - Forma: transcripción y } \\
\text { sonidos extranjerizantes. } \\
\text { - Morfología inestable. } \\
\text { - Monosemia. Sin conno- } \\
\text { tación. } \\
\text { - Marcas autonímicas. } \\
\text { - Uso circunstancial (cam- } \\
\text { po de especialidad). }\end{array}$ & $\begin{array}{l}\text { - Forma: asimilación gráfico- } \\
\text { fónica. } \\
\text { - Morfología más estable. } \\
\text { - Abandono de la monosemia. } \\
\text { Connotación; especialización } \\
\text { semántica. } \\
\text { - Marcas autonímicas (dismi- } \\
\text { nución). } \\
\text { - Uso regular (apenas se sien- } \\
\text { te exógeno). }\end{array}$ & $\begin{array}{l}\text { - Asimilación total en } \\
\text { grafía, sonido y morfo- } \\
\text { logía. } \\
\text { - Generación: morfolo- } \\
\text { gía y significados. } \\
\text { - Uso regular (percibi- } \\
\text { do como patrimonial). }\end{array}$ \\
\hline
\end{tabular}

se ha considerado que cualquier frecuencia debía ser calificada de «circunstancial» 0 «no significativa»".

8 Aunque el factor "tiempo de entrada" sea fundamental, a veces no resulta del todo determinante. Así, leemos en Gómez CAPUz (2005, p. 15): "El propio criterio cronológico puede fallar. A este respecto, podemos citar la incompleta integración de club y sandwich, dos de los anglicismos más antiguos del español (principios del siglo XIX): a pesar de su antigüedad, presentan bastantes variaciones en su pronunciación (/klu/, / klub/), las cuales se agudizan en el ámbito morfológico de la formación del plural: clubs, clus y clubes, sandwiches y sandwichs". De este modo, el tiempo de introducción será considerado, pero no determinará la inclusión de un elemento léxico en uno u otro grupo: únicamente las pruebas lingüísticas y no las extralingüísticas, que serán coadyuvantes (como "el tiempo" o "el valor" o "uso general” de los usuarios), serán las que establezcan su cualidad. 
JAPONESISMOS DE LA RELIGIÓN Y LA FILOSOFÍA

«bonzo»

Según concluimos en Fernández Mata 2018, el término podría proceder de los étimos 凡僧 ([bonso: $]$ o [bonzo: $]^{9}$ ) o 梵僧 ([bonso: $]$ ), ambos inventariados en el Daiyirín, que define el sustantivo 凡僧 con las siguientes acepciones en japonés moderno: “(1) Monje de rango inferior que no ha sido nombrado $S \bar{g} g \bar{o}$ (antigua autoridad eclesiástica budista); (2) Monje común”. En cuanto a 梵僧, posee estos descriptores semánticos: “(1) Monje que se consagra a la austeridad y la pureza y obedece los preceptos religiosos; (2) Monje; (3) Monje hindú". Aunque el Daiyirín únicamente recoja el furigana con sibilante sorda, そう [so:], en el Daiyisén Digital ${ }^{10}$ aparece el furigana ぼんぞう-articulado [bonzo:] - en una de las definiciones de 凡僧, que traducimos a continuación: “(1) [igual que bonzo] rango inferior a Sōgō (antigua autoridad eclesiástica budista), o monje que no tiene el cargo o la responsabilidad de $S \bar{g} g \bar{o}$, monje en la categoría de $B \bar{o} s h i$ (monje budista); (2) Monje común”.

A pesar de que el CORDE cuente con un solo ejemplo de grafía arcaica, bonços, lo cierto es que en los corpus (CORDE, CREA y CORPES XXI) hay uniformidad en cuanto a la representación de la palabra, que aparece siempre como bonzo(s). Por lo que respecta a las obras lexicográficas españolas (DEA, DLE 2001, GDUEA, DUEAE, DUE, DClave y DLE 2014), también detectamos uniformidad: concuerdan con el significante preferido en los corpus. Únicamente hemos encontrado la transcripción extranjerizante bonze en la obra de Bluteau (1721), inventariada por el NTLLE. Coincide esta grafía con la empleada en inglés $(M W C D$ y $O E D)$ y francés $(D F L$ y $L P R)$. Por su parte, el portugués (DHLP y DPLP) y el italiano (DOVLI y Zingarelli) presentan igual significante que el español, esto es, bonzo. Sobre su acentuación, en lengua española no hay lugar a dudas, tanto por la información fónica descrita en los diccionarios GDUEA y DClave como por la ausencia de tildes en las muestras de los corpus, se observa que este vocablo ha sido adaptado con patrón llano.

En lo referente a su significado, hay consenso entre los corpus (CORDE, CREA y CORPES XXI) del español y las obras lexicográficas

${ }^{9}$ En cuanto a la transcripción fonética que llevamos a cabo en este trabajo, ensombreceremos los segmentos vocálicos que, según nuestra percepción, se articulan con mayor intensidad. Nos hemos basado en las muestras de hablantes nativos reales contenidas en el diccionario de pronunciación en línea: http://es.forvo.com/. - [z]: sonido coronal, fricativo o africado, sonoro (AKAMATSU 1997, pp. 95-96; VANCE 2008, p. 88).

${ }_{10}$ Diccionario del japonés moderno, también disponible en el portal en línea Kotobank (www.kotobank.jp). 
españolas (DLE 2001, GDUEA, DUEAE, DUE, DClave y DLE 2014), que refieren con la forma bonzo a 'un monje o sacerdote budista'. En otras lenguas romances, existen acepciones peyorativas. Así, en francés $(D F L$ y $L P R)$ e italiano (DOVLI, Zingarelli) también puede significar 'persona pretenciosa o que se da aires de superioridad'. Esta misma acepción la recoge el DHLP para el portugués, lengua en la que, además, se observan otros valores semánticos, como el de 'hipócrita' y ‘perezoso' (DHLP y DPLP). Aunque el español actual no cuente con estas acepciones, en una de las obras lexicográficas recogidas en el NTLLE, la de Castro y Rossi (1852, s.v.), hallamos la siguiente caracterización de los bonzos, por la cual se intuye que en épocas pretéritas en español éstos también podían ser considerados como 'perezosos' u 'holgazanes':

Según los vocabularios consultados, en la China y otras tierras de gentiles, se distinguen con este nombre los que profesan una vida al parecer austera, y viven separados de la gente común, en conventos ó desiertos. Esta clase de gente está á cargo de la sociedad por una costumbre antigua, que ha pasado á constituirse ley: el comercio atiende á su sustento por un derecho voluntario sobre los géneros de importación y de esportación, el cual, aunque módico, reúne á la conclusión del año cantidades exorbitantes, y fomenta, por decirlo así, la holgazanería de esta gente.

Asimismo, gracias al CORDE, encontramos en algunos extractos de la Crónica de la provincia de San Gregorio Magno en las Islas Filipinas (1676), de fray Francisco de Santa Inés, descripciones por las que los castellanos tachan a los bonzos de 'hipócritas' y 'codiciosos':

Parece que se juntó en estos bonzos del Japón toda la hipocresía de los fariseos; porque mirando su compostura exterior y su blandura en el hablar y tratar con todos, parecen hombres de gran santidad y virtud, y son los más viciosos y llenos de pecados que hay en aquella tierra.

...y éste es uno de los mayores impedimentos que hay en aquellos reinos para la predicación del Evangelio, porque viendo los bonzos cuán contrario es lo que en él se enseña á sus pecados y á la hipocresía de su vida, y que con él se descubre la falsedad de sus sectas, de lo cual resulta perder sus devotos feligreses y disminuirse en gran parte sus rentas y limosnas, procuran con todas sus fuerzas que nadie le reciba, ni haya quien le predique, poniendo todos los medios posibles para estorbarlo.

...la pobreza voluntaria; que aunque no la reverenciaba como evangélica, por no saber las cosas de nuestra Fe, teníala por virtud moral, religiosa y ejercitada aquí en extremo grado de perfección, más que la que usan sus bonzos. Entre éstos, aquéllos son tenidos por más perfectos, que son más pobres, más penitentes y que más desprecian las honras del mundo; aunque nunca llega á ser tal su pobreza, desprecio y penitencia, 
que tenga asomos de virtud; antes sí una hipocresía fina, de la cual se valen para ocultar su codicia y ambición y otros muchos vicios.

Aparte de estos casos fortuitos y pretéritos, el valor semántico mayoritario reflejado por los corpus es el de 'monje o sacerdote budista'. De ahí que el japonesismo aparezca constantemente en contextos donde se mencionan aspectos religiosos, sobre todo en las primeras muestras del CORDE, pertenecientes a textos de misioneros: cristiandad, Nuestro Señor, idolos, templos, conventos, monasterios, Cielo, predicar, secta, sacerdotes, etcétera.

Debido a su referente masculino no resulta extraño que, en su integración en el sistema morfológico del español, los corpus (CORDE, CREA y CORPES XXI) y los diccionarios españoles (DLE 2001, GDUEA, DUEAE, DUE, DClave y DLE 2014) coincidan en describirlo como sustantivo masculino ${ }^{11}$. Así lo demuestran los numerosísimos ejemplos de los corpus, en que viene acompañado por elementos adyacentes masculinos, tanto en singular ( $e l, u n, a l$, del, algún), como plural (los, algunos, nuestros, muchos, estos mismos). Con este género ha pasado a las otras lenguas romances que lo utilizan: el francés (DFL y $L P R)$, el portugués (DHLP y DPLP) y el italiano (DOVLI y Zingarelli).

Mención especial merece la locución adverbial a lo bonzo. Según las obras lexicográficas hispánicas, ésta se puede usar junto a los verbos quemarse [a lo bonzo] (DEA, DLE 2001, GDUEA, DUEAE, DUE, DClave, DLE 2014) o autoinmolarse/ prenderse fuego [a lo bonzo] (DUEAE) para constituir una combinación sintáctica que indica que 'una persona se ha rociado de líquido inflamable y se ha prendido fuego en público en acción de protesta o solidaridad'. Si bien en el CREA se muestra preferencia por la construcción con el verbo suicidarse [a lo bonzo], y el CORPES XXI recoge igualmente la combinación inmolarse [a lo bonzo], a veces, también puede funcionar como locución adjetiva junto a sustantivos como suicidio [a lo bonzo] (DEA, DLE 2001, DLE 2014) o pelo [a lo bonzo] (GDUEA) -aunque esta última indica 'cabeza rasurada'. En el CREA hallamos otros ejemplos con diferentes sustantivos: autoinmolación/ resistencia [a lo bonzo]. De acuerdo con los datos del CREA, esta locución se empleó por primera vez en un artículo del diario El Pais, publicado el 2 de febrero de 1977. Las obras lexicográficas extranjeras ( $M W C D, O E D, D F L, L P R, D H L P, D P L P, D O V L I y$ Zingarelli) no recogen una expresión similar, por lo que inferimos que esta locución es propiamente española.

11 Sin embargo, durante el proceso de adopción de la palabra, encontramos testimonios que acreditan una forma y uso para el femenino. Gracias al NTLLE, hallamos en la obra de Terreros y Pando (1786, s.v.): "asimismo hai Bonzas, que son las mujeres, ó hijas de los Bonzos, y viven en comunidad, y como en una especie de Monasterios". En francés, el LPR indica una forma femenina anticuada: bonzesse. 
Dependiendo de la fuente, contamos con dos fechas para el primer registro escrito de «bonzo» en español. El DCELC de Corominas (1954, p. 487) data la forma bonzo en 1618, sin especificar la fuente, pero gracias al CORDE podemos adelantar su aparición a 1583, en la Vida de San Ignacio de Loyola escrita por Pedro de Ribadeneira, quien emplea el plural bonzos.

En lo que concierne a la lengua portuguesa, según Dalgado (1919, p. 138), la primera referencia se halla en plural (bonzos) en 1545, en el cap. 78 de la Peregrinação de Fernão Pinto, publicada póstumamente en 1614. Si consideramos como fortuitas excepciones las grafías bôzos, bouzos (de 1578) ${ }^{12}$ y bonzes (del siglo XVI), desde los primeros testimonios inventariados por Dalgado (1919, pp. 138-139), se observa que en portugués, al igual que en español, su transcripción también es estable, en bonzo(s). El Zingarelli sostiene que el japonesismo en lengua italiana ha sido tomado del portugués bonzo, y que el año de su primera aparición se remonta a 1549. En el $L P R$ también se cree que la forma bonze del francés actual deriva de la portuguesa bonzo, y fecha el primer registro escrito en francés en 1570. En cuanto al inglés, los primeros datos que se tienen de su forma actual (bon$z e$ ), procedente de la francesa, son más tardíos (1618 -boze- y 1688 -bonzes-, según el $O E D ; 1653$-no se especifica la forma- en $M W C D$ ), si bien el $O E D$ indica que algunos de los primeros registros en inglés parecen derivar directamente del étimo japonés. En 1552 se recoge el significante bonzii, y en 1588, bonsos. La primera surge de la latinización del étimo japonés, concretamente la forma plural de la segunda declinación. La segunda, bonsos, más parece proceder del español o del portugués. Se observa, por tanto, que todas las lenguas consultadas dan como intermediaria la portuguesa ${ }^{13}$.

Para concluir, creemos que la voz bonzo, con distribución panhispánica, se encuentra totalmente asimilada, esto es, forma parte de las voces patrimoniales. Hay pruebas que demuestran lo anterior: grafía, patrón acentual y morfológico firmemente establecidos, sin lugar a variaciones; significación estable que puede dar lugar incluso a locuciones idiomáticas, que ya vimos en párrafos precedentes. A pesar de que en sus primeros registros existen marcas autonímicas para entender su significado (que assi se llaman entre ellos sus sacerdotes y sabios; muchos sacerdotes de idolos, a quien llaman bonzos; religiosos a quien llaman bonzos; o religiosos de la China; que son sus sacerdotes; ó sacerdote de los gentiles; etc.), lo cierto es que ni en CREA ni en CORPES XXI se detectan. Su repercusión, según el CREA, es baja (0.48 casos

12 Respecto de estos dos casos, Dalgado (1919, p. 138) afirma que el "Padre Luís Fróis, muito competente no assunto, escreve mais de uma vez Bouzos, e para que não houvesse ambiguìdade com respeito a $u$ por $n$, ortografa em um passo Bôzos"; es decir, que las grafías bôzos y bouzos son equivalentes a bonzo, con nasal.

13 Para la resolución de esta incógnita, véase Fernández Mata 2018. 
por millón para la forma bonzo), mientras que en el CORPES XXI es circunstancial o no significativa (0.08 casos por millón).

TABLA 2

Distribución geográfica de bonzo

\begin{tabular}{|c|c|c|c|c|c|}
\hline \multicolumn{3}{|c|}{ CREA } & \multicolumn{3}{|c|}{ CORPES XXI } \\
\hline País & Freq & Fnorm & País & Freq & Fnorm \\
\hline España & 56 & 0.75 & España & 11 & 0.12 \\
\hline Colombia & 2 & 0.43 & Argentina & 3 & 0.13 \\
\hline México & 2 & 0.16 & Colombia & 1 & 0.05 \\
\hline Perú & 2 & 0.52 & Cuba & 1 & 0.11 \\
\hline Argentina & 1 & 0.07 & El Salvador & 1 & 0.27 \\
\hline \multirow[t]{3}{*}{ Estados Unidos } & 1 & 0.41 & Guinea Ecuatorial & 1 & 1.08 \\
\hline & & & México & 1 & 0.03 \\
\hline & & & Venezuela & 1 & 0.08 \\
\hline
\end{tabular}

«en»

La voz procede del étimo 禅 [zen], que en japonés posee dos acepciones: “(1) Abreviatura de la transcripción de dhyāna (禅那 senna) que puede traducirse como calma permanente. Término budista. Concentrar la mente y entrar en un estado en el que no existe el yo; (2) Abreviatura de budismo «cen»" (DaiD).

Tanto en los corpus (CORDE, CREA y CORPES XXI) como en las obras lexicográficas españolas (DEA, DLE 2001, GDUEA, DUEAE, DUE, DClave y DLE 2014) y extranjeras (MWCD, OED, DFL, LPR, DHLP, $D P L P, D O V L I$ y Zingarelli), se utiliza la transcripción extranjerizante zen. La grafía $z$ - puede articularse como $s$ dental en el español meridional, o como interdental, fricativa, $[\theta]$, en la modalidad minoritaria septentrional (GDUEA). Sobre su acentuación, en lengua española no hay lugar a dudas al tratarse de un sustantivo monosilábico.

Por lo que se refiere a su carga semántica, en los corpus del espanol (CORDE, CREA y CORPES XXI) observamos dos significados según la categoría gramatical. Con función de sustantivo, los diccionarios españoles (DEA, DLE 2001, GDUEA, DUEAE, DUE, DClavey DLE 2014) lo definen como

forma / escuela / doctrina / práctica / sistema filosófico de budismo o budista que, mediante técnicas de meditación de carácter metafísico con las que evitan los esquemas conceptuales y detienen el curso del pensamiento, pretende alcanzar la iluminación: la esencia de la verdad. 
Como adjetivo, 'aplicado a esta forma / escuela / doctrina / práctica o sistema filosófico' ${ }^{14}$. Apréciese cómo el valor semántico del español es una mezcla de los dos descriptores semánticos usados en lengua japonesa (DaiD).

El primitivo sustantivo «cen», utilizado de forma pospuesta, comenzó a emplearse con función adjetiva en lengua española. Las obras lexicográficas hispánicas registran ambos usos: como sustantivo, todas concuerdan en describirlo con género masculino (DEA, DLE 2001, GDUEA, DUEAE, DUE, DClave y DLE 2014), y así aparece registrado en los ejemplos de los corpus, junto a adyacentes como $\mathrm{el}$, $\mathrm{un}$, al, del, japonés, puro, propio, etc. En función de adjetivo es invariable (DEA) y se coloca pospuesto a un sustantivo (DClave): rockero zen, temperamento zen, monje zen, límite zen, simplicidad zen, añagaza zen, literatura zen, planteamientos zen, prácticas zen, reminiscencias zen, etc. No encontramos casos de plural, aunque sí detectamos las siguientes combinaciones léxicas: zen japonés, budismo zen, maestro zen, pensamiento zen, monje zen y jardín zen.

En español, según el CORDE, el primer registro escrito data de 1963. Su primera aparición en una obra lexicográfica española tiene lugar en el DEA, esto es, en 1999. Desde su registro inicial no observamos variación formal o significativa.

En las otras lenguas analizadas, su primer registro escrito es mucho anterior. En inglés, el $M W C D$ y el $O E D$ concuerdan en datarlo en 1727. El LPR lo sitúa en 1889 para la lengua francesa. En italiano,

TABLA 3

Distribución geográfica de zen

\begin{tabular}{|c|c|c|c|c|c|}
\hline \multicolumn{3}{|c|}{ CREA } & \multicolumn{3}{|c|}{ CORPES XXI } \\
\hline Pais & Freq & Fnorm & País & Freq & Fnorm \\
\hline España & 194 & 2.63 & España & 171 & 2.01 \\
\hline Argentina & 55 & 4.30 & Argentina & 58 & 2.67 \\
\hline Cuba & 44 & 13.66 & Chile & 47 & 3.29 \\
\hline México & 15 & 1.21 & México & 46 & 1.70 \\
\hline Venezuela & 6 & 1.38 & Colombia & 19 & 1.10 \\
\hline Chile & 5 & 0.91 & Venezuela & 9 & 0.75 \\
\hline Colombia & 3 & 0.65 & Perú & 8 & 1.07 \\
\hline \multirow[t]{3}{*}{ Perú } & 2 & 0.52 & Paraguay & 7 & 1.28 \\
\hline & & & República Dominicana & 7 & 1.22 \\
\hline & & & Costa Rica & 4 & 1.27 \\
\hline
\end{tabular}

14 En el resto de lenguas estudiadas funciona de manera similar. 
según información del DOVLI y del Zingarelli, su primer testimonio escrito corresponde a 1929.

A partir de la valoración de todos los datos anteriores, creemos que la voz «cen» se encuentra en el grupo de japonesismos que han sido casi totalmente asimilados por la lengua española. No podemos afirmar que se trata de una voz patrimonial, pues, por un lado, su transcripción es extranjerizante y, por otro, su referente significativo es todavía percibido como elemento exógeno (encontramos marcas autonímicas del tipo japonés o zen japonés). Además, en función de adjetivo, no presenta concordancia con el sustantivo al que define, si bien, tanto en CREA como en CORPES XXI, presenta una frecuencia baja (2.43 casos por millón en el primero y 1.61 casos por millón en el segundo) en las naciones hispánicas.

«sintoísmo»

La lexía «sintoísmo» proviene de la voz 神道 [s’into: ${ }^{15}$, que en japonés cuenta con las dos siguientes acepciones:

(1) Práctica religiosa tradicional del pueblo japonés, así como las ideas y la actitud vital que la sostienen. Surgió de prácticas animistas y chamanistas, para ir centrándose progresivamente en la adoración a los antepasados, dioses del clan y los dioses padres de la patria hasta ser instituida como culto nacional por la corte imperial Iamato. A pesar de ser un desarrollo independiente al de religiones traídas del exterior, como el budismo, más tarde fue recibiendo la influencia tanto de éste como del confucianismo, dando lugar a distintas teorías del sintoísmo; (2) Referido al budismo, herejía en la que se deposita la fe en dioses demonio (Daiyirín).

Al significante sinto se añadió el sufijo /-ismo/, cuya primera función, según el DLE 2014, es la de formar "sustantivos que suelen significar doctrinas, sistemas, escuelas o movimientos".

Observamos variedad de representación gráfica en la comparación de los corpus, causada por la articulación extranjerizante del elemento japonés [s'i], que a veces se articula como prepalatal, fricativa, [S], y, otras, según la lengua española, [s] (GDUEA y DClave). En COR$\mathrm{DE}$, se muestra preferencia por sintoísmo (5 casos, 1 con mayúscula inicial: Sintoísmo) frente a Shinto (1 caso). En CREA, se continúa esta tendencia: mayoría de sintoísmo(s) (51 casos, 3 con mayúscula inicial: Sintoísmo) frente a shinto (18 casos, 7 con mayúscula inicial: Shinto) y shintoísmo (11 casos). La variedad escrita llega hasta el CORPES XXI,

15 [s'] sonido coronal, palatalizado, fricativo, sordo (AкAмAтsu 1997, pp. 91-92; VANCE 2008, pp. 78, 81). 
pero en él los casos de Shinto (4) superan a los de sintoísmo (3) y shintoísmo (1). Las obras lexicográficas españolas únicamente inventarían la fórmula sintoísmo (DEA, DLE 2001, GDUEA, DUEAE, DUE, DClave y DLE 2014), lo cual contrasta con algunas obras lexicográficas extranjeras, que sí registran el uso sinonímico de sus dos significantes: Shinto/ Shintoism (MWCD y OED), shintoïsme/ shinto (DFL y LPR), xintó / xintoísmo / sintoísmo (DHLPy DPLP). En italiano no se emplea la forma shinto, pero, por el funcionamiento interno de su ortografía frente al extranjerismo, sí encontramos variedad gráfica en scintoismo/ shintoismo (DOVLIy Zingarelli). Por lo que respecta al uso de la mayúscula inicial, la RAE, en su Ortografía (2010, p. 496), indica que "tanto los sustantivos que designan las distintas religiones como los que designan al conjunto de los fieles de una religión son nombres comunes y deben escribirse con minúscula inicial". En la composición de sinto + ismo, el acento recae sobre el sufijo español, de lo que resulta una palabra paroxítona, como así podemos ver en las muestras de los corpus y en la descripción fonética del GDUEA y el DClave.

En lo referente a su valor semántico, las obras lexicográficas hispánicas lo describen como "religión primitiva de origen japonés que se caracteriza por ser politeísta, animista-mitológica -adoración de las fuerzas naturales-y por presentar culto a los antepasados" (DEA, DLE 2001, GDUEA, DUEAE, DUE, DClave y DLE 2014). En lo básico, las obras lexicográficas extranjeras concuerdan con la definición de las hispánicas, aunque añaden dos aspectos que no se recogen en español: "(1) veneración / exaltación de la figura del Emperador, descendiente de la diosa Sol” (MWCD, OED, LPR, DOVLIy Zingarelli); “(2) esta religión es anterior al budismo" (DFL, DPLPy Zingarelli). Apreciamos, pues, que la definición de los diccionarios hispánicos deriva de la primera acepción del Daiyirín para el japonés. A pesar de que nuestros diccionarios no describen con la amplitud de las obras lexicográficas extranjeras el término, en los corpus sí detectamos un uso que abarca todos los semas nombrados, por lo que el japonesismo suele aparecer junto a vocablos como religión indígena, impuros, budismo, confucionismo, teológica, Mikado, emperador, diosa Amaterasu, mística, rezo, antepasados, animismo, dioses, etcétera.

Las obras lexicográficas de la lengua española (DEA, DLE 2001, GDUEA, DUEAE, DUE, DClave y DLE 2014) lo describen como sustantivo masculino y así aparece en los corpus (CORDE, CREA y CORPES XXI), junto a elementos adyacentes que indican su género: $e l$, un, al, del, antiguo, sincrético, tántrico, caracterizados, etc. En otras lenguas romances, este japonesismo también ha pasado como sustantivo masculino (DLF, LPR, DHLP, DPLP, DOVLI, Zingarelli). Con tan sólo un caso en CREA, no podemos afirmar que su forma plural, sintoísmos, se use con tanta regularidad como la singular, aunque debemos tener en cuenta que los significantes plurales de las religiones no se 
emplean de manera generalizada; no se suele utilizar: los cristianismos, los budismos, los hinduismos, etc., por el referente de colectividad y pluralidad que ya viene indicado en el sufijo /-ismo/. No detectamos preferencia por ningún patrón combinatorio, aunque sí se registra un lexema derivado de sinto: sintoista "perteneciente o relativo al sintoísmo / partidario del sintoísmo" (adj. o sust. común DVUA, DEA, DLE 2001, GDUEA, DUEAE, DUE, DClave y DLE 2014).

Contamos con varias fechas para la datación de su primer registro escrito. De acuerdo con los datos del CORDE, la primera documentación textual de sintoísmo en español comprende los años que van de 1953 a 1974, mientras que Shinto se fecha en 1964. Podemos adelantar su registro gracias a la información del NTLLE, donde descubrimos que la obra de Gaspar y Roig, de 1855, recoge las formas Sinto y Sintoísmo. Desde su primer registro escrito, no encontramos cambios semánticos importantes, ni formales.

En otras lenguas europeas, su primera documentación es muy anterior. En portugués, por ejemplo, el $D H L P$ data la forma seutó en 1612 y shintoismo en 1874. En inglés, por su parte, se registra Sinto en 1727 (MWCD y $O E D)$ y Sintooism en $1857(O E D)$. El LPR sitúa shintoïsme en 1765. Por último, DOVLI y Zingarelli fechan el primer registro escrito de scintoismo o shintoismo-no se especifica la grafía- en 1905.

\section{TABLA 4}

Distribución geográfica de sintoísmo - shinto

\begin{tabular}{|c|c|c|c|c|c|}
\hline \multicolumn{3}{|c|}{ CREA } & \multicolumn{3}{|c|}{ CORPES XXI } \\
\hline \multicolumn{3}{|l|}{ (sintoísmo) } & \multicolumn{3}{|l|}{ (sintoísmo) } \\
\hline País & Freq & Fnorm & País & Freq & Fnorm \\
\hline \multirow[t]{4}{*}{ España } & \multirow[t]{4}{*}{52} & \multirow[t]{4}{*}{0.70} & España & 2 & 0.02 \\
\hline & & & Chile & 1 & 0.07 \\
\hline & & & Paraguay & 1 & 0.18 \\
\hline & & & Puerto Rico & 1 & 0.25 \\
\hline \multicolumn{3}{|c|}{ (shinto o Shinto) } & \multicolumn{3}{|l|}{ (shinto o Shinto) } \\
\hline País & Freq & Fnorm & País & Freq & Fnorm \\
\hline España & 9 & 0.12 & Argentina & 3 & 0.13 \\
\hline Argentina & 5 & 0.39 & Ecuador & 1 & 0.17 \\
\hline México & 2 & 0.16 & España & 1 & 0.01 \\
\hline \multirow[t]{4}{*}{ Colombia } & 1 & 0.21 & Guatemala & 1 & 0.27 \\
\hline & & & Nicaragua & 1 & 0.28 \\
\hline & & & Puerto Rico & 1 & 0.25 \\
\hline & & & República Dominicana & 1 & 0.17 \\
\hline
\end{tabular}


Teniendo en cuenta la información de los párrafos precedentes, consideramos que la voz «sintoísmo» pertenece al grupo de los japonesismos que se encuentran en proceso de asimilación. Hay muestras que prueban su inestabilidad en el sistema: variación gráfica o transcripción extranjerizante y valor semántico monosémico, que en ocasiones necesita explicarse por marcas autonímicas (significaba camino de los dioses; que combinaba el culto de los antepasados con creencias en espiritus y en las fuerzas naturales; la religión tradicional; se centraba en el culto al emperador, considerado como descendiente de la diosa Amaterasu; es la religión nacional del Japón, formada por creencias y ritos ancestrales, centrados en la adoración de fuerzas sobrenaturales denominadas Kami y que presenta una diversidad zonal y temporal muy notable que aconseja su estudio siguiendo un esquema histórico, etc.). Según el CREA, su frecuencia normalizada es baja, 0.39 casos por millón para sintoísmo -sólo registrado en España- y no significativa, de 0.12 casos por millón para shinto/ Shinto-España, Argentina, México y Colombia. El CORPES XXI indica que la tendencia a usarse de manera circunstancial se ha extendido en todo el territorio hispánico, tanto para la forma sintoísmo -con 0.02 casos por millón- como para shinto/ Shinto -con 0.03 casos por millón.

\section{«zacén»}

El préstamo «zacén» proviene del étimo japonés, con doble posibilidad gráfica, 座禅 o 坐禅 [zazen], definido como "uno de los principales métodos de estudio para alcanzar el conocimiento dentro del budismo, en especial, método de concentración que es considerado el núcleo del budismo cen" (Daiyirín).

Mientras que en CREA se prefiere la grafía extranjerizante zazen ( 5 casos), sin marcas acentuales, frente a los 2 casos de la forma extranjerizante, pero con marcas acentuales, zazén, el CORPES XXI muestra preferencia por esta última: 4 casos de zazén vs. 1 caso de zazen. En las obras lexicográficas, española (DClave) o extranjeras (OED, DFL y Zingarelli), se utiliza el significante zazen. Las muestras acentuadas en los corpus y la descripción fónica del DClave indican que en español ha sido adoptada como voz aguda, al igual que el étimo japonés.

En lo que respecta a su significado, el DClave lo describe como "práctica de meditación budista que consiste en dejar la mente en blanco y concentrarse en la respiración". Las obras lexicográficas extranjeras definen de manera similar el término (OED, DFL y Zingarelli). Por tanto, derivan directamente de la acepción japonesa (Daiyirín). En las muestras de los corpus, el japonesismo suele aparecer junto a términos relacionados con su definición, como practicar, mente, meditación budista zen, sentado, sentarse, templo, satori, pensadores religiosos, etcétera. 
El DClave indica que se trata de un sustantivo masculino, y así aparece registrado en los corpus (CREA y CORPES XXI), junto a adyacentes del tipo el, del, mucho. En francés (DFL) e italiano (Zingarelli -invariable-) también se emplea con género masculino. No hallamos muestras de plural, aunque sí algunas combinaciones recurrentes, como practicar(se) «zacén», sentar(se) / sentado en (posición de) «zacén».

La primera documentación lexicográfica que se tiene de esta voz la hace el DClave, por lo tanto, hemos de fecharla en 2012. Sin embargo, gracias al CREA, podemos adelantar su primer registro a 1993 . Desde su primera aparición no encontramos cambios formales o significativos. En otras lenguas, su primera documentación es anterior: el $O E D$ fecha su primer registro en 1727, y el Zingarelli, para el italiano, en 1983.

Por los datos recién expuestos, consideramos que esta voz se encuentra en proceso de transferencia, ya que presenta grafía extranjerizante, con aplicación o no de las reglas de acentuación, y cuenta con un único significado, aplicado a un elemento cultural exógeno, que en ocasiones aparece definido mediante marcas autonímicas del tipo meditación budista zen, meditación en postura Zen, meditación sentada, etc. En CREA, su repercusión es circunstancial (0.03 casos por millón para zazen), tendencia que continúa en el CORPES XXI (0.02 casos por millón para zazén y zazen). En ambos corpus observamos que su distribución geográfica es panhispánica.

TABLA 5

Distribución geográfica de zazen / zazén

\begin{tabular}{|c|c|c|c|c|c|}
\hline \multicolumn{3}{|c|}{ CREA } & \multicolumn{3}{|c|}{ CORPES XXI } \\
\hline País & Freq & Fnorm & País & Freq & Fnorm \\
\hline España & 3 & 0.04 & Colombia & 5 & 0.29 \\
\hline Argentina & 1 & 0.07 & Estados Unidos & 2 & 0.62 \\
\hline México & 1 & 0.08 & & & \\
\hline
\end{tabular}

\section{Conclusiones}

Después de sopesar los datos de los párrafos anteriores, podemos extraer las siguientes conclusiones:

1) Casi la totalidad de las voces examinadas no presenta variación articulatoria de carácter segmental; únicamente «sintoísmo», cuya sibilante prepalatal en ocasiones queda transcrita con el dígrafo $s h$, y, otras, la mayoría, con $s$. Asimismo, en su adaptación al español, observamos preferencia, como no podía ser de otro modo, por el patrón acentual paroxítono, pues la mitad de las voces han sido 
adaptadas como palabras llanas ( «bonzo» ${ }^{16} \mathrm{y}$ «sintoísmo» ${ }^{17}$ ). Sigue el patrón acentual del étimo la voz «zacén», de carácter oxítono, mientras que «cen», por ser un elemento monosilábico, queda obviamente

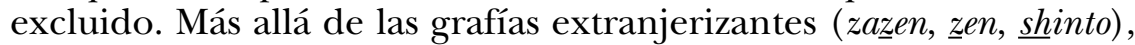
que pueden deberse al influjo del sistema de romanización Hepburn -de origen anglosajón-, no tenemos constancia de que otras lenguas hayan intermediado en el proceso de adopción entre el étimo japonés y la adaptación hispánica.

2) El análisis morfosintáctico de las voces nos revela que el $100 \%$ pertenece a la categoría de los sustantivos, todos con género masculino ${ }^{18}$. Con el japonesismo «bonzo» ha surgido una locución adverbial de naturaleza hispánica, pues no se detecta en otras lenguas próximas: a lo bonzo ${ }^{19}$. A veces, también puede funcionar como locución adjetiva junto a sustantivos como suicidio / autoinmolación / resistencia [a lo bonzo] o pelo [a lo bonzo]. En cuanto a «zacén», hallamos algunas combinaciones recurrentes. No ocurre así con «sintoísmo», aunque sí se registra un lexema derivado de sinto: sintoísta, con función adjetiva o de sustantivo común. Por último, el sustantivo «cen», pospuesto a los sustantivos ${ }^{20}$, posee función adjetiva.

3) En lo concerniente al plano semántico, la totalidad de las voces mantiene semejanza con el/los significado/s original/es de los étimos. Atendiendo a los valores semánticos de las cuatro voces, una («bonzo») es usada para denominar a una clase de 'monje budista', mientras que el resto hace referencia a elementos abstractos: «sintoísmo» 'religión', «cen» 'doctrina filosófica', «zacén» 'práctica de meditación'.

4) Un análisis cronológico revela que la mitad de los japonesismos religioso-filosóficos se introdujo en la lengua española a lo largo del siglo xx (1963: «cen»; 1993: «zacén»). En cambio, «sintoísmo», cuyo primer registro se fecha en 1855 , fue adoptado durante lo que convinimos en llamar la etapa de aislamiento de Japón. Por último, debemos incluir la voz «bonzo», cuya primera documentación data de $1583^{21}$, dentro del selecto grupo de japonesismos introducido en la

${ }^{16}$ Esta unidad ha sido adaptada con el mismo patrón paroxítono que la lengua japonesa.

17 Téngase presente que, en la composición de sinto + ismo, el acento recae sobre el sufijo español.

${ }^{18}$ Recuérdese que hemos descubierto usos pretéritos de «bonzas».

19 Según el CORPES XXI, en la actualidad su distribución geográfica es panhispánica. No obstante, la horquilla de años que separa la primera documentación española, en 1977, y la chilena (1984) o venezolana (1989), nos hace pensar que esta construcción pudiera ser fruto de España (cf. CREA).

${ }^{20}$ En el apartado correspondiente pueden observarse las combinaciones sintácticas que hemos encontrado.

${ }^{21}$ Se trata, hasta que otras investigaciones muestren lo contario, del japonesismo usado en la actualidad con una documentación más antigua. 
primera etapa de contacto (1580-1619) entre los pueblos hispánicos y Japón ${ }^{22}$.

5) Si tenemos en cuenta la repercusión y distribución de estos japonesismos en el sistema léxico de la lengua española actual, todas las voces comparten un uso panhispánico: tres vocablos se caracterizan por una frecuencia circunstancial («sintoísmo», «zacén»y «bonzo» ${ }^{23}$ ), mientras que «cen» posee una frecuencia baja.

6) Según el grado de adaptación al sistema léxico del español, un elemento ha pasado a formar parte de las voces patrimoniales: «bonzo», en tanto que «cen» casi alcanza una asimilación total. Por su parte, «sintoísmo» se encuentra en proceso de adaptación, esto es, en el segundo estadio de adopción, y «zacén», en pleno proceso de transferencia, por lo que todavía se considera extranjerismo.

TABLA 6

Estadio de los japonesismos analizados

\begin{tabular}{lll}
\hline $1^{a}$ etapa & $2^{a}$ etapa & $3^{a}$ etapa \\
Momento de transferencia & Momento de asimilación & Momento de madurez \\
\hline «zacén» & $\begin{array}{l}\text { «intoísmo» } \\
\text { «cen» }\end{array}$ & «bonzo» \\
\hline
\end{tabular}

7) Para concluir, en relación con los dos puntos anteriores, nos preguntamos si el parámetro "tipología textual", es decir, la frecuencia de uso de un vocablo en un tipo de texto, podría utilizarse para determinar la etapa de adopción de los préstamos. En el CORPES XXI detectamos que el total de los japonesismos analizados en este artículo presenta como tipología textual mayoritaria la ficción.

A priori, podría considerarse que, para que una voz se utilice en un texto ficcional, ésta ha debido ser asimilada completamente por la gran masa de hablantes, ya que, en principio, un autor escribe para ser comprendido. Además, hacer uso de un vocablo "foráneo", "marginal", "de introducción reciente", implica que el autor antes ha debido interiorizarlo de algún modo. Sin embargo, contrastando nuestros datos, observamos que, si bien las cuatro voces se emplean principalmente en textos ficcionales, sólo dos, «bonzo»y «cen», se encuentran en la tercera etapa-adopción completa- o muy cerca de ella. ¿Debemos, pues, tener en cuenta el parámetro de la tipología textual a la hora de establecer el momento de adopción de un préstamo? De

${ }^{22}$ Para la etapa de aislamiento y la primera etapa, véase Fernández Mata 2017, pp. 162-164.

${ }^{23}$ Los ordenamos de menor a mayor, según la frecuencia normalizada. 
TABLA 7

Frecuencia tipológica de las voces

\begin{tabular}{llcc}
\hline & Tipología & Freq & Fnorm. \\
\hline bonzo & Ficción & 6 & 0.07 \\
& Noticia & 1 & 0.08 \\
zen & Ficción & 133 & 1.77 \\
& Reportaje & 16 & 3.09 \\
& Divulgación & 15 & 1.13 \\
& Académico & 10 & 0.73 \\
& Blog & 8 & 2.14 \\
& Noticia & 5 & 0.41 \\
& Crítica & 5 & 6.90 \\
& Entrevista digital & 2 & 18.08 \\
& Biografía/memoria & 1 & 1.10 \\
zazen & Opinión & 1 & 0.53 \\
zazén & & & \\
sintoísmo & Ficción & 5 & 0.06 \\
Shinto & Ficción & 3 & 0.03 \\
shinto & Noticia & 4 & 0.33 \\
\hline
\end{tabular}

acuerdo con nuestra investigación, este factor no resulta del todo fiable por tres motivos:

1) el carácter subjetivo del creador literario. Si un autor, verbigracia mi caso, está familiarizado con la lengua prestataria (el japonés), le resultará más fácil, e incluso natural, encajar la voz en cualquier enunciado de su obra.

2) Asimismo, en la redacción de su trabajo, el autor puede tomarse licencias poéticas o creativas que no tengan en consideración si la voz empleada es entendida o no por los lectores.

3) Otro factor que invalidaría dicho parámetro es la escasez de muestras: que uno o dos autores utilicen cierta voz no es síntoma de que ésta haya sido completamente adoptada -tal vez se dé la coincidencia de que ambos conozcan en cierto sentido la lengua prestataria-, si bien este hecho podría iniciar el proceso de adopción.

Por tanto, estimamos que, para que este parámetro resulte efectivo, debemos combinarlo con el resto de factores y, además, valorar la cantidad de autores que utilizan el préstamo, pues, a mayor número de casos registrados, mayor número de autores-creadores que conocen la palabra y que, con cuyo empleo, demuestran la integración de la voz en el sistema léxico de la lengua. 


\section{REFERENCIAS}

Akamatsu, Tsutomu 1997. Japanese phonetics: Theory and practice, LINCOM Europa, München.

Almarza, Nieves et al. 2012. Diccionario Clave: diccionario de uso del español actual, S.M., Madrid.

Alvar, Manuel et al. 1967. Enciclopedia lingüistica hispánica, t. 2, Consejo Superior de Investigaciones Científicas, Madrid.

Alvar EzQuerra, Manuel 2012 [1994], La formación de palabras en español, Arco/ Libros, Madrid.

Alvar Ezquerra, Manuel (dir.) 1994. Diccionario de voces de uso actual, Arco/Libros, Madrid.

Alvar EzQuerra, Manuel (dir.) 2003. Nuevo diccionario de voces de uso actual, Arco/ Libros, Madrid.

Cannella, Mario e Beata Lazzarini (dirs.) 2014. Lo Zingarelli 2015: Vocabolario della lingua italiana, Zanichelli, Bolonia.

Cid Lucas, Fernando 2009. "La presencia de palabras japonesas en el castellano: una lectura antropológica de su incorporación”, Observatorio de la Economía y la Sociedad del Japón, 1, 6.

CORDE = Real Academia Española: Banco de datos (CORDE) [en línea]. Corpus diacrónico del español, http://www.rae.es [consultado el 11 de agosto de 2017].

Corominas, JoAn 1954. Diccionario crítico etimológico de la lengua castellana, Francke, Berna.

CORPES XXI = Real Academia Española: Banco de datos (CORPES XXI) [en línea]. Corpus del español del Siglo XXI (CORPES), http://www.rae.es [consultado el 11 de agosto de 2017].

CREA = Real Academia Española: Banco de datos (CREA) [en línea]. Corpus de referencia del español actual, http://www.rae.es [consultado el 11 de agosto de 2017].

Dalgado, Sebastião Rodolfo 1919. Glossário luso-asiático, t. 1, Imprensa da Universidade, Coímbra.

Devoto, Giacomo e Gian Carlo Oli 2012. Il Devoto-Oli: vocabolario della lingua italiana 2013, Le Monnier, Firenze. [CD-ROM].

Diccionario de la Real Academia Española 2001. Real Academia Española-Espasa Calpe, Madrid. [CD-ROM].

Dicionário Priberam da língua portuguesa 2008-2013. Priberam Informática [en línea], http:// www.priberam.pt/ dlpo/ [consultado el 11 de agosto de 2017].

$D L E=$ RAE-ASALE 2014. Diccionario de la lengua española 2014 . 23a ed., Real Academia Española-Espasa Libros, Madrid, en línea http://www.rae.es/ [consultado el 11 de agosto de 2017].

Fernández Mata, RAFAel 2015. Los japonesismos de la lengua española: historia y transcripción, tesis, Universidad Pablo de Olavide, https://rio.upo.es/ xmlui/ handle/ 10433/3716 [consultada el 11 de agosto de 2017].

FERnÁndez Mata, RAFAel 2016. "Etiqueta para denominar los préstamos léxicos de la lengua japonesa: japonesismos”, Español Actual, 105, pp. 55-69.

Fernández Mata, Rafael 2017. "Los japonesismos del español actual”, Revista de Filología de la Universidad de La Laguna, 35, pp. 149-168.

Fernández Mata, RAFAel 2018. "El origen etimológico del japonesismo bonzo", Cuadernos del Instituto Historia de la Lengua, 11, pp. 29-44.

Fernández Mata, Rafael 2018a. "Método de transcripción del japonés al español: los sonidos vocálicos, semivocálicos y consonánticos”, Onomázein, 42, pp. 237-276.

Frago Gracia, Juan Antonio 1997. "Japonesismos entre Acapulco y Sevilla: sobre biombo, catana y maque”, Boletín de Filología de la Universidad de Chile, 36, pp. 101118. 
García Yebra, Valentín 1994. Traducción: historia y teoría, Gredos, Madrid.

GIL, JUAN 1991. Hidalgos y samuráis: España y Japón en los siglos XVI y XVI, Alianza Editorial, Madrid.

Giménez Folqués, David 2012. "Los extranjerismos en el español académico del siglo xxI”, Normas. Revista de Estudios Lingüísticos Hispánicos, anejo 3, pp. 1-79.

Gómez Capuz, Juan 1998. El préstamo lingüístico: conceptos, problemas y métodos. Cuadernos de Filología, Universitat de València, València.

Gómez Capuz, Juan 2004. Préstamos del español: lengua y sociedad, Arco/Libros, Madrid.

Gómez Capuz, Juan 2005. La inmigración léxica, Arco/Libros, Madrid.

Houaiss, Antônio (dir.) 2001. Dicionário Houaiss da língua portuguesa, Editora Objectiva, Rio de Janeiro.

Jeuge-Maynart, Isabelle (dir.) 2016. Dictionnaire de français Larousse, Hachette Livre-Département Informatique Groupe Livre (DSI), Paris, http:// www. larousse.fr/ dictionnaires/ francais-monolingue [consultado el 11 de agosto de 2017].

Kim, Tai Whan 1992. "Análisis lingüístico de los japonesismos en Triunfo de la fee en los reynos de Japón, de Lope de Vega”, Actas del II Congreso Internacional de Historia de la Lengua Española, Pabellón de España, Madrid, t. 1, pp. 1355-1358.

Knowlton, Edgar Colby 1959. Words of Chinese, Japanese, and Korean origin in the Romance languages, Stanford University, Stanford.

Lahuerta Galán, Javier (dir.) 2003. Diccionario de uso del español de América y Espa$\tilde{n} a$, Vox, Barcelona. [CD-ROM].

Martinet, André 1991 [1965]. Elementos de lingüistica general, Gredos, Madrid.

Matsumura, Akira (dir.) 2006. Daiyirín, Sanseido, Tokyo [en línea], www.kotobank. jp [consultado el 11 de agosto de 2017].

Matsumura, Akira (dir.) 2008. Daiyisén Digital, Sanseido, Tokyo [en línea], www. kotobank.jp [consultado el 11 de agosto de 2017].

Merriam-Webster's Collegiate Dictionary 2003. $11^{\mathrm{a}}$ ed., Merriam-Webster, Springfield. [CD-ROM].

Moliner, María 2008. Diccionario de uso del español, Gredos, Madrid. [Ed. en CDROM basada en la $3^{\text {a }}$ ed. en papel de 2007].

$N T L L E=$ Real Academia Española, Nuevo Tesoro Lexicográfico de la Lengua Española (NTLLE) [en línea], http://ntlle.rae.es/ ntlle/ SrvltGUILoginNtlle [consultado el 11 de agosto de 2017].

Ortografía de la lengua española 2010. Real Academia Española-Espasa, Madrid.

Prieto Vera, Luis 2007. "Voces de origen japonés en el léxico de la prensa de Santiago de Chile", Boletín de Filología, 42, pp. 157-318.

Rey, Alain (dir.) 2014. Le Petit Robert de la langue française, Le Robert, Paris. [CDROM].

Reyes Díaz, María Josefa 2004. “A propósito de léxico y cultura”, en Traducción, lenguas, literaturas. Sociedad del conocimiento. Enfoques desde y hacia la cultura. Ed. Sonia Bravo Utrera, Universidad de Las Palmas de Gran Canaria, Las Palmas de Gran Canaria, pp. 221-233.

Robins, Robert Henry 1995 [1971]. Lingüistica general. Estudio introductorio, Gredos, Madrid.

SAla, MARIUs 1998 [1986]. Lengua en contacto, Gredos, Madrid.

SÁnchez, AQuilino 2006 [2001]. Gran diccionario de uso del español actual, SGEL, Madrid. [CD-ROM].

SÁnchez, AQuilino et al. 1995. Cumbre. Corpus lingüístico del español contemporáneo, SGEL, Madrid.

Seco, Manuel, Olimpia Andrés y Gabino Ramos 1999. Diccionario del español actual, Aguilar, Madrid.

Simpson, John (dir.) 2009. Oxford English dictionary, Oxford University Press, Oxford. [En CD-ROM, basada en la $2^{\mathrm{a}}$ ed. en papel de 1989]. 
Vance, Timothy J. 2008. The sounds of Japanese, Cambridge University Press, Cambridge.

WeINREICH, Uriel 1974 [1968]. Lenguas en contacto: descubrimientos y problemas. Pref. de André Martinet. Trad. de Francisco Rivera, Biblioteca de la Universidad Central de Venezuela, Caracas.

\section{ANEXO: LISTADO DE ABREVIAGIONES EMPLEADAS}

$\begin{array}{ll}\text { CORDE } & \text { Corpus diacrónico del español } \\ \text { CORPES XXI } & \text { Corpus del español del siglo XXI } \\ \text { CREA } & \text { Corpus de referencia del español actual } \\ \text { DaiD } & \text { Daiyisén Digital } \\ \text { Daiyirín } & \text { Daiyirín } \\ \text { DClave } & \text { Diccionario Clave: diccionario de uso del español actual } \\ \text { DCELC } & \text { Diccionario crítico etimológico de la lengua castellana } \\ \text { DEA } & \text { Diccionario del español actual } \\ \text { DFL } & \text { Dictionnaire de Français Larousse } \\ \text { DHLP } & \text { Dicionário Houaiss da Lingua Portuguesa } \\ \text { DLE } & \text { Diccionario de la Lengua Española } \\ \text { DPLP } & \text { Dicionário Priberam da Lingua Portuguesa } \\ \text { DOVLI } & \text { Devoto-Oli: vocabolario della lingua italiana } \\ \text { DUE } & \text { Diccionario de uso del español } \\ \text { DUEAE } & \text { Diccionario de uso del español de América y España } \\ \text { DVUA } & \text { Diccionario de voces de uso actual } \\ \text { GDUEA } & \text { Gran diccionario de uso del español actual } \\ \text { LPR } & \text { Le Petit Robert de la langue française } \\ \text { MWCD } & \text { Merriam-Webster's Collegiate Dictionary } \\ \text { NDVUA } & \text { Nuevo diccionario de voces de uso actual } \\ \text { NTLLE } & \text { Nuevo Tesoro Lexicográfico de la Lengua Española } \\ \text { OED } & \text { Oxford English Dictionary } \\ \text { Zingarelli } & \text { lo Zingarelli 2015. Vocabolario della lingua italiana } \\ & \end{array}$

